Acta Cryst. (1954). 7, 477

\title{
A Suggested Explanation of the Occurrence of Forbidden X-ray Reflexions in the $\sigma$-Phase
}

\author{
By J. A. BLand \\ Crystallographic Laboratory, Cavendish Laboratory, Cambridge, England
}

(Received 20 March 1954)

\begin{abstract}
'Forbidden reflexions' in the $\mathrm{Ni}-\mathrm{V} \sigma$-phase reported by previous workers have been confirmed. Their explanation, which requires ordering in the structure, is discussed and an alternative explanation, based on the Renninger 'double reflexion' effect, is proposed. The conditions for the occurrence of the double reflexions are examined and are shown to be particularly favourable in the present problem.
\end{abstract}

\section{Introduction}

Pearson \& Christian (1952) investigated the $\sigma$-phase in the vanadium-nickel system and found a tetragonal unit cell with $a_{0}=8.966 \AA$ and $c_{0}=4 \cdot 641 \AA$. They compared observed structure factors with those calculated from the $\beta$-uranium and cobalt-chromium $\sigma$ phase structures and showed that vanadium-nickel $\sigma$-phase is isomorphous with them. In the course of this work they recorded with chromium radiation several reflexions which are among the systematic absences demanded by the space group $(P 4 / m n m$, $P 4 n m$, or $P \overline{4} n 2$ ). These reflexions were not observed with copper radiation.

\section{Repetition of single-crystal experiments}

The experiments have been repeated in this laboratory using material kindly supplied by Pearson \& Christian. Two crystals of approximate dimensions $0.2 \mathrm{~mm}$. were irradiated with chromium, molybdenum and copper radiations while oscillating about their [010] axes. The following $h 0 l$ (or $0 k l$ ) reflexions with $h+l$ (or $k+l$ ) odd, and which therefore break the spacegroup rule, were observed with chromium radiation only. Those observed by Pearson \& Christian are also listed.

$\begin{array}{cc}\text { Present work } & \text { Pearson \& Christian } \\ 00 \overline{1} & 001 \\ 601 & 003 \\ 60 \overline{1} & 601 \\ 20 \overline{3} & 021 \\ 0 \overline{2} \overline{3} & \end{array}$

All the reflexions in the left-hand column were fairly sharp and of low intensity. $00 \overline{1}$ could be obtained easily but the others were not reproducible. The $0 \overline{23}$ reflexion on the second layer line was observed only in the lower half of the photograph.

\section{Interpretation}

(1) Ordering in the structure

It was originally suggested by Pearson \& Christian that the $\sigma$-phase in the nickel-vanadium system is partly ordered, and that these additional reflexions are due to the superlattice. On this view, their intensity will depend on wavelength owing to the 'anomalous dispersion' of X-rays by atoms. The frequency of the molybdenum radiation is so far removed from the absorption edges of vanadium and nickel that their scattering factors are unaffected and the difference between them, for $\sin \theta / \lambda=0$, will be 5 . The frequency of chromium radiation is so close to the absorption edge of vanadium that $f_{V}$ is reduced by 3 , and the difference of scattering factors is thus increased from 5 to 8 . With copper radiation on the other hand the difference is diminished to about $1 \cdot 5$. Thus the extra spots are expected to be strongest for chromium, less strong for molybdenum (but the geometrical factor will be more favourable than with chromium), and almost zero for copper radiation. In this way the absence of the effect with copper radiation was explained.

\section{(2) Double reflexions}

An alternative explanation, proposed here, is that the forbidden reflexions arise from the 'Renninger effect', i.e. the double reflexion of X-rays from two sets of 'strong' planes (Renninger, 1937). The forbidden reflexion $h k l$ may be simulated if the $\mathrm{X}$-rays reflected from a plane $h_{1} k_{1} l_{1}$ are scattered in turn from $h_{2} k_{2} l_{2}$. (We shall call $h_{1} k_{1} l_{1}$ the generating reflexion and $h_{2} k_{2} l_{2}$ the associated reflexion.) The conditions which must be fulfilled are:

(a) The Bragg law must be satisfied for two sets of planes simultaneously, namely $h k l$ and $h_{1} k_{1} l_{1}$. This means that for a given setting of the crystal both reciprocal lattice points must lie on the reflecting sphere. Moreover, the rays scattered from $h_{1} k_{1} l_{1}$ must serve as incident beam for scattering from $h_{2} k_{2} l_{2}$. Hence

$$
h=h_{1}+h_{2} ; k=k_{1}+k_{2} ; l=l_{1}+l_{2} .
$$

(b) Both $h_{1} k_{1} l_{1}$ and $h_{2} k_{2} l_{2}$ reflexions must be strong. 
When these conditions are satisfied $h_{1} k_{1} l_{1}$ and a weak sharp spot, which will be indexed as $h k l$, will be observed on the same film. $h_{1} k_{1} l_{1}$ and $h_{2} k_{2} l_{2}$ must, of course, be consistent with the space-group though no such condition is placed on $h k l$.

The following is a list of reflexions which, with chromium radiation, satisfy conditions $(a)$ and $(b)$ and give rise to the observed 'forbidden' diffraction orders :

\begin{tabular}{|c|c|c|c|c|}
\hline Forbidden & \multicolumn{2}{|l|}{ Generating } & \multicolumn{2}{|c|}{ Associated } \\
\hline$h k l$ & $h_{1} k_{k_{1}} l_{1}$ & & $h_{2} k_{2} l$ & \\
\hline $00 \overline{\mathbf{l}}$ & $720 \quad b, v s$ & $\begin{array}{l}\text { with } \\
\text { or }\end{array}$ & $\overline{721}$ & $b, v s$ \\
\hline & $22 \overline{2} \quad s$ & with & 221 & $v s$ \\
\hline 601 & $72 \overline{1} \quad b, v s$ & $\begin{array}{l}\text { with } \\
\text { or }\end{array}$ & $\overline{12} 2$ & $v s$ \\
\hline & $11 \overline{2} s$ & with & $5 \overline{1} 3$ & $b, v s$ \\
\hline $\begin{array}{l}601 \\
20 \overline{3}\end{array}$ & $\begin{array}{ll}513 & b, v s \\
41 \overline{3} & b, v s\end{array}$ & $\begin{array}{l}\text { with } \\
\text { with }\end{array}$ & $\frac{1}{2} 10$ & $\begin{array}{l}s \\
s\end{array}$ \\
\hline $0 \overline{\mathbf{2 3}}$ & $5 \overline{\mathbf{1}} \overline{3} \quad b, v s$ & with & $\overline{510}$ & $s$ \\
\hline
\end{tabular}

For the most part the generating reflexions were observed to be broadened. This eharacteristic is associated with high Bragg angle $\left(\sim 85^{\circ}\right)$. Hence condition $(a)$ is not as stringent as might be expected, the tolerance being about $\pm 1^{\circ}$. In particular this explains why $00 \overrightarrow{1}$ is easily reproducible, for both $h_{1} k_{1} l_{1}(720)$ and $h_{2} k_{2} l_{2}(\overline{72} \overline{1})$ are broadened and very strong.

Even though broadening does make the condition less stringent it can be shown that the maximum effect should in general be obtained when the crystal is slightly mis-set in that its [010] axis does not coincide exactly with the rotation axis. The amount of missetting required for $(a)$ to be precisely satisfied for each observed forbidden reflexion was found to be of the order of $\frac{1}{2}^{\circ}$. It is proposed that $00 \overline{3}$ and $02 \overline{1}$, which were observed by Pearson \& Christian but not in the present work, may have been produced by the following double reflexions, for each of which a missetting greater than $\frac{1}{2}^{\circ}$ is required:

$\begin{array}{ccc}\text { Forbidden } & \text { Generating } & \text { Associated } \\ h k l & h_{1} k_{1} l_{1} & h_{2} k_{2} l_{2} \\ 00 \overline{3} & \overline{5} \overline{3} & \overline{5} \overline{1} 0 \\ 02 \overline{1} & 41 \overline{3} & \overline{4} 12\end{array}$

To confirm this, it would be necessary to have two films showing $(00 \overline{3}, 51 \overline{3})$ and $(02 \overline{1}, 41 \overline{3})$ respectively.

Finally, it was shown that the forbidden reflexions recorded with chromium radiation cannot be prcduced with molybdenum or copper radiations because pairs of planes do not then exist which satisfy conditions $(a)$ and $(b)$.

\section{Discussion of the two interpretations}

The two interpretations are best compared by considering the observations which they have to explain. They are:

(1) Forbidden reflexions are observed with chromium radiation.

(2) They are not observed with copper radiation.

(3) They are not observed with molybdenum radiation.

(4) The reflexions are elusive, i.e. they cannot always be reproduced. Spots observed by Pearson \& Christian were not obtained here, and vice-versa.

(5) When the reflexion is not on the zero layer line it is seen in the upper or lower half of the film but not in both at the same time.

The ordering explanation adequately accounto for only (1) and (2). It also predicts that the retiexions should be produced by molybdenum radiation, which contradicts (3).

The second interpretation, in terms of the Renninger effect, accounts for all five observations.

In conclusion it may be stated that there is no reliable evidence for ordering in the nickel-vanadium $\sigma$-phase.

I should like to thank Dr W. H. Taylor and Dr A. M. B. Douglas for their encouragement and helpful discussions.

\section{References}

Pearson, W. B. \& Christian, J. W. (1952). Acta Cryst. $5,157$.

Renninger, M. (1937). Z. Phys. 106, 141. 